\title{
Connaissances, Attitudes et Comportements des mères d'enfants malnutris aigus modérés sur la gestion des ordures ménagères
}

\section{Knowledge, Attitudes and Behaviors of mothers of moderately acutely malnourished children on household waste management}

Sow Nouhoum ${ }^{1}$, Konaté Fatoumata ${ }^{1}$, Sangho Fanta ${ }^{1,2^{*}}$, Sangho Aboubacar ${ }^{3}$, Sangho Oumar ${ }^{1,2}$, Coulibaly Cheick Abou ${ }^{1}$, Traoré Mohamed Moumine $^{1}$, Coulibaly Djénéba ${ }^{1,2}$, Touré Fady ${ }^{4}$, Telly Nouhoum ${ }^{1,5}$, Berthé Mamadou ${ }^{6}$, Diawara Fatou ${ }^{1,2,6}$, Diallo Baba ${ }^{7}$, Kéïta Salia ${ }^{1}$, Diarra Moussa Modibo ${ }^{8}$, Sangho Fadimata ${ }^{9}$,Touré Ousmane ${ }^{1,10}$

1. Département d'Enseignement et de Recherche en Santé Publique et Spécialités, Faculté de Médecine et d'OdontoStomatologie (FMOS) / Université des Sciences, des Techniques et des Technologies de Bamako (USTTB), Mali

2. Département d'Enseignement et de Recherche des Sciences Biologiques et Médicales, Faculté de Pharmacie (FAPH), USTTB, Mali

3. Département d'Enseignement et de Recherche des Sciences Pharmaceutiques, FAPH/USTTB, Mali

4. Programme National de lutte contre le Paludisme (PNLP), Bamako, Mali

5. Cellule Sectorielle de Lutte Contre le SIDA la Tuberculose et les Hépatites Virales (CSLS-TBH), Bamako, Mali

6. Institut National de Santé Publique (INSP), Bamako, Mali

7. Centre Hospitalier Universitaire, Centre National d'OdontoStomatologie (CNOS), Bamako, Mali

8. Centre de Santé de Référence (CSRéf) de Kéniéba, Kayes, Mali

9. Cellule de Planification et de Statistique, Secteur Santé, Développement Social et Promotion de la Famille (CPS/SS-DSPF), Bamako, Mali

10. Institut National de Formation en Sciences de la Santé (INFSS) Bamako, Mali

*Auteur correspondant : Dr Fanta Sangho, Maître-Assistant en Santé Communautaire, Département d'Enseignement et de Recherche des Sciences Biologiques et Médicales (DERSBM), FAPH, USTTB. Email : sanghofanta@gmail.com, Tél : +2236678 2728

\section{RESUME}

Introduction : La malnutrition est l'un des principaux problèmes de santé et de bien-être qui affectent les enfants dans les pays en développement en général et au Mali en particulier. Elle peut être répandue même lorsque la disponibilité alimentaire est suffisante à cause des habitudes alimentaires, des us et coutumes ; mais également à cause des comportements qui favorisent la prolifération des vecteurs biologiques, sources de contamination des aliments. L'accumulation des ordures ménagères, quelle qu'en soit la cause, porte préjudice à la santé. Elles peuvent être à l'origine de la prolifération de microbes, et autres vecteurs de maladies. Selon l'Organisation Mondiale de la Santé (OMS), l'insalubrité de l'environnement tue chaque année 3 millions d'enfants dans le monde. L'objectif était d'étudier les connaissances, attitudes et comportements des mères sur la gestion des ordures ménagères. Méthodologie : il s'agissait d'une étude transversale descriptive par sondage simple des mères ou tutrices d'enfants souffrant de malnutrition aiguë modérée, résidant dans le quartier de Yirimadio en commune VI du district de Bamako, qui s'est déroulée de décembre 2017 à janvier 2018. Résultats : cette étude a révélé que $62 \%$ des mères ont utilisé la poubelle pour stocker leurs ordures ménagères ; la voie publique a été utilisé dans $94 \%$ pour éliminer les ordures. L'incinération à ciel ouvert a été utilisée dans $6 \%$ de cas ; $59 \%$ des enquêtées ont versé les eaux usées dans les rues. Conclusion : Cette situation nécessite des solutions efficaces et durables, d'où la nécessité de mise en place des stratégies pour une gestion durable des ordures ménagères dans le quartier de Yirimadio incluant l'information et la sensibilisation.

\section{ABSTRACT}

Introduction: Malnutrition is one of the major health and well-being issues affecting children in developing countries in general and Mali in particular. It can be widespread even when food availability is sufficient because of dietary habits, habits and customs; but also because of behaviors that promote the proliferation of biological vectors, sources of food contamination. The accumulation of garbage, whatever the cause, is detrimental to health. They can be the cause of the proliferation of microbes, and other vectors of diseases. According to WHO, unhealthy environments kill 3 million children every year worldwide. Our study aimed to study the knowledge, attitudes and behaviors of mothers on the management of household waste. Methods: It was a descriptive cross-sectional survey of mothers or guardians of children suffering from moderate acute malnutrition, residing in the Yirimadio district in Commune VI of the Bamako district and ran from December 2017 to January 2018. Results: This study revealed that $62 \%$ of mothers used the trash to store their household waste; public roads were used in $94 \%$ to eliminate garbage. Open burning was used in $6 \%$ of cases; $59 \%$ of the respondents poured the wastewater into the streets. Conclusion: This situation requires effective and sustainable solutions, hence the need to put in place strategies for sustainable management of household waste in the Yirimadio district, including information and awareness.

Keywords: Malnutrition, household waste, waste

\section{Introduction}

Selon la définition de l'Organisation Mondiale de la Santé (OMS), la malnutrition se caractérise par un " état pathologique résultant de la carence ou de l'excès, relatif ou absolu, d'un ou plusieurs nutriments essentiels, que cet état se manifeste cliniquement ou ne soit décelable que par des analyses biochimiques, anthropométriques ou physiologiques». Elle résulte aussi bien d'une alimentation inadéquate que d'un environnement sanitaire déficient. 
Les pratiques alimentaires inadéquates font références, non seulement à la qualité et à la quantité des aliments donnés aux enfants, mais aussi aux étapes de leur introduction (1)

La malnutrition est l'un des principaux problèmes de santé et de bien-être qui affectent les enfants dans les pays en développement y compris le Mali.

Elle peut être répandue même lorsque la disponibilité alimentaire est suffisante à cause des habitudes alimentaires, des us et coutumes qui privent certains membres de la communauté d'un apport alimentaire adéquat ; mais également à cause des comportements qui favorisent la prolifération des vecteurs biologiques, sources de contamination des aliments.

L'OMS estime que 159 millions d'enfants de moins de cinq ans sont trop petits pour leur âge (atteints de retard de croissance) ; 50 millions ne pèsent pas assez pour leur taille (émaciés) et 41 millions sont en surpoids ; aucun de ces enfants ne se développe sainement (2). Dans 14 pays, moins de la moitié des enfants de moins de cinq ans échappent au retard de croissance et à l'émaciation. La malnutrition est responsable de 2,6 millions de morts d'enfants chaque année soit un tiers des décès dans le monde (2).

Les prévalences de sous nutritions en Afrique de l'Ouest sont parmi les plus élevées du monde. Le retard de croissance touche $39 \%$ des enfants de moins de 5 ans, soit 27 millions d'enfants de moins de 5 ans dans la région. Environ $12 \%$ des enfants de moins de cinq ans - soit 7.8 millions d'enfants- souffrent de malnutrition aiguë globale (MAG). Dans 8 pays de la région, la prévalence de la MAG est égale ou supérieure à $10 \%$, ce qui est le seuil d'alerte selon les standards de l'OMS.

Le Mali est l'un des pays les plus pauvres au monde, classé 174ème sur 177 pays selon l'Indice de Développement humain des Nations Unies, avec un revenu par tête d'habitant de l'ordre de 380 dollars américains en 2005 (1). Les niveaux d'alphabétisation restent parmi les plus bas au monde, avec ( $81 \%$ ) d'adultes et $(76 \%)$ de jeunes analphabètes (3). Au Mali, 38\% des enfants souffrent de malnutrition chronique contre $19 \%$ sous la forme sévère. Le niveau du retard de croissance augmente rapidement avec l'âge. II est de $15 \%$ chez les enfants de moins de 6 mois, passe à $24 \%$ chez ceux de 9 11 mois, puis continue d'augmenter pour atteindre un maximum de $48 \%$ à $18-23$ mois, pour se maintenir à un niveau élevé après cet âge (4). Les résultats de l'EDSM IV (en 2006) montrent qu'au Mali, (191) enfants pour mille décèdent avant leur 5 ème anniversaire. A cela, il faut ajouter que $(43 \%)$ d'entre eux souffrent de retard de croissance (malnutrition chronique), (15\%) d'émaciation et $(27 \%)$ d'insuffisance pondérale (1). La prévalence nationale de la malnutrition aiguë globale (MAG) s'élève à $13,3 \%$ (1).

Dans le district de Bamako, cette prévalence est proche du seuil critique qui est $15 \%$. Ces données confirment la gravité de la situation nutritionnelle (1). En effet, selon la classification de l'OMS, le seuil d'alerte est fixé à $10 \%$.
Bien que la malnutrition soit de plus en plus un problème de développement, le phénomène est beaucoup plus ressenti dans les zones périurbaines ou la majeure partie de la population à un niveau socio-économique faible.

Dans le district sanitaire de la commune VI en particulier le quartier de Yirimadio, quartier périurbaine, la malnutrition reste une préoccupation majeure de la population et la tendance de la prévalence de la malnutrition s'avère inquiétante, malgré les efforts entrepris par l'Etat et ses différentes Partenaires.

La malnutrition fait partie des 5 principales causes de mortalité infantile dans la commune. La malnutrition est à la fois une cause et une conséquence majeure de la pauvreté, de l'insécurité alimentaire et de l'inégalité sociale. Elle est à l'origine non seulement d'une grande vulnérabilité à l'infection et à d'autres troubles, mais aussi des handicaps intellectuels, mentaux, sociaux et de développement. Elle est aussi une conséquence de la mauvaise gestion des ordures ménagères (5).

Depuis des années, nous avons vu que la plus grande partie du village de Yirimadio est menacée par la mauvaise gestion des ordures de tout genre, dont les ordures ménagères sont à la base de l'insalubrité. Cette insalubrité est causée par l'homme lui-même parce qu'il jette volontairement des ordures dans les rues et dans des parcelles le long des routes.

L'environnement physique du quartier de Yirimadio subit des menaces causées par l'augmentation de la population qui s'élève aujourd'hui à 17418 habitants (DNSI 2008). Pendant nos observations à Yirimadio, nous avons remarqué que la population connaît aujourd'hui des problèmes de santé, ayant pour conséquence directe la dégradation de l'état nutritionnel des enfants de moins cinq ans, dus à la mauvaise gestion des ordures ménagères. Dans les rues de Yirimadio, l'insalubrité s'observe par la présence des sachets, des déchets des cuisines, des boites de conserve éparpillées le long des chemins et parcelle, les excréments des animaux domestiques et autres sur lesquels pullulent les mouches et les moustiques.

Toutes ces raisons nous ont incité à réaliser cette étude sur les connaissances, attitudes et pratiques des mères et tutrices d'enfants de Yirimadio sur la gestion des ordures ménagères afin de comprendre le lien avec la survenue de la malnutrition et pouvoir proposer des suggestions et recommandations pour améliorer l'efficacité des interventions des programmes de survie de l'enfant. L'objectif était de décrire les connaissances, les attitudes et les pratiques des mères ou tutrices d'enfants souffrant de malnutrition aiguë modérée (MAM) en matière de gestion des ordures ménagères.

\section{Méthodologie \\ Cadre de l'étude}

L'étude s'est déroulée au Centre de Santé Communautaire de Yirimadio, sis dans ledit quartier. Le quartier de Yirimadio est situé en Commune VI du District de Bamako. II a une population de 17418 habitants (DNSI 
2008). Il est limité à l'Est par le village de Niamana, au Nord par Missabougou, au Sud par le village de Sirakoro Méguétana et à l'Ouest par Banankabougou Faladié (6). Le climat est tropical, de type soudanais caractérisé par une saison sèche (décembre-mai) et une saison pluvieuse (juin-novembre) qui correspond à la haute transmission du paludisme. La population est composée de Bambara, Peulh, Sonrhaï, Dogon, Sarakolé, Senoufo, Mianka, Bobo etc. La langue la plus parlée est le Bambara (6). L'agriculture est la principale source de revenu des ménages suivie de l'élevage d'ovins et de caprins et du commerce. La commune VI connaît l'un des plus forts taux de croissance du District de Bamako et compte parmi les plus pauvres de ses communes. Le taux de pauvreté est très élevé : $83,6 \%$ (7). La précarité économique des ménages se caractérise par la pratique de micro-activités (petit artisanat, revente, recyclage, collecte de gravats, petit maraîchage, transport inter urbain), permettant des rentrées d'argent irrégulières et faibles (6). II est à noter également que de mauvaises conditions d'hygiène sanitaire et alimentaire sont dues à l'insuffisance d'infrastructures et d'ouvrages d'assainissement de base. Ce qui peut augmenter la fréquence de certaines affections telles que les maladies diarrhéiques, les infections respiratoire aiguë, le paludisme, la malnutrition etc. (7).

Type d'étude :

II s'agissait d'une étude transversale descriptive par sondage simple des mères ou tutrice d'enfants souffrant de malnutrition aiguë modérée, résidant dans le quartier de Yirimadio en commune VI du district de Bamako.

Période d'étude :

Elle s'étendait de Décembre 2017 à Janvier 2018.

Population d'étude :

La population cible était constituée des enfants de 6 à 59 mois, des mères et tutrices d'enfants résidant à Yirimadio au moment de l'enquête.

\section{Critère d'inclusion :}

- Mères et tutrices d'enfants de 6 à 59 mois souffrant de malnutrition aiguë modérée ;

- Résider, au moins 6 mois précédant l'enquête, à Yirimadio ;

- Donner son consentement.

Critère de non inclusion :

- Mères ou tutrices d'enfants souffrant d'autres pathologies d'urgence ;

- Mères ou tutrices pressées ou n'ayant pas le temps pour l'interview.

Taille de l'échantillonnage et échantillonnage :

La taille de l'échantillon a été calculée selon la formule suivante (OMS, 1991) :

$$
\mathrm{N}=\mathrm{P} Q /[\mathrm{E} / L]^{2} \quad \mathrm{~N}=\mathrm{P}(1-\mathrm{P}) /[\mathrm{E} / 1,96]^{2}
$$

Avec $\mathrm{N}$ : taille minimale de l'échantillon nécessaire ; $P$ : estimation de la proportion attendue (taux de prévalence) ; $Q$ : la valeur de (1-P) ; E : Marge d'erreur tolérée (risque statistique de $5 \%$ ) ; $L$ : écart réduit pour le risque statistique admis (1,96 pour le risque $5 \%$ ).
En considérant la prévalence de la malnutrition de $15 \%$ au niveau national (EDSM V), $\mathrm{N}=216$.

Nous avons effectué un échantillon aléatoire simple.

Technique et outils de collecte des données :

Les entretiens ont été réalisés avec l'aide d'un questionnaire en mode face à face.

Gestion et analyse des données :

La saisie des données a été réalisée dans le logiciel EPIINFO version 6.04 et analysées sur le logiciel EXCEL 2010. Les données ont été présentées dans des tableaux avec calcul des proportions.

Considérations éthiques et déontologiques :

Le consentement oral des parents a été obtenu après des explications sur l'étude et son intérêt. L'accord des responsables administratifs et techniques du centre a été reçu. La confidentialité des informations recueillies leur a été garantie.

\section{Résultats}

Caractéristiques sociodémographiques de la population étudiée (tableau I) :

La classe d'âge [26 - 34[ans était la plus fréquente. L'âge moyen était de 34 ans. Plus de la moitie $(51,9 \%)$ était mariée. La moitié $(50,5 \%)$ des ménages n'était pas scolarisée. Environ $44 \%$ des mères étaient des ménagères.

Connaissances, attitudes et comportements des mères :

Au total $93 \%$ des mères avaient entendu parlé de la malnutrition.

Les 201 mères enquêtées soit 93\% affirmaient que la mauvaise gestion des ordures ménagères peut provoquer des maladies. Le paludisme et la diarrhée étaient les maladies, causées par l'insalubrité, citées respectivement par $80 \%$ et $20 \%$.

Selon les mères, la maigreur était le signe clinique le plus fréquent avec $\mathbf{4 5 , 4 \%}$ (tableau II). Les maladies ont été signalées par $54,2 \%$ des répondants, comme conséquence de la mauvaise gestion des ordures ménagères (tableau II).

La majorité des femmes soit $62 \%$ utilisaient des poubelles à domicile contre $38 \%$ qui n'en utilisaient pas.

Parmi les femmes enquêtées, $37 \%$ déversaient les ordures ménagères dans les rues et $26,9 \%$ sur la route (tableau II). La majorité $(58,8 \%)$ déversait les eaux usées dans les rues (tableau II).

\section{Discussion}

Au cours de notre étude nous avons enquêté 216 mères d'enfants âgés de 06-59 mois sur un total de 382 enfants de 06-59 mois détectés malnutris aigus modérés dans les ménages par les Agents de Santé Communautaire (ASC), d'où une prévalence de $3,5 \%$ (6). Ce résultat était supérieur à celui de l'INSTAT dans une étude en $2016 \mathrm{au}$ Mali qui trouve 1,2\% de prévalence de MAM à Bamako (8). Ceci pourrait s'expliquer par le fait que Yirimadio est une zone périurbaine, avec un niveau de revenu de la 
population relativement faible. Au Kenya la prévalence était de 6,3\% (9).

Caractéristiques socio démographiques des mères d'enfants malnutris aigus modérés :

Parmi les mères des enfants dépistés MAM, la tranche d'âge de 26 à 34 ans était la plus représentée avec 30,1\%, ce résultat est différent de celui trouvé par NANTOUME I (10) qui avait trouvé 58,4\% pour la tranche d'âge de 21 à 35 ans. Plus de la moitié des mères étaient mariées $(51,9 \%)$. Ce résultat est similaire à celui de KEMESSO B (11) lors d'une enquête menée dans le quartier de Yirimadio.

Dans notre étude les mères non scolarisées représentaient $50,5 \%$. Ce résultat était similaire à celui de NANTOUME I (10) à Yirimadio 49,7\% et supérieur à celui de Djiré (12) qui trouve $14,1 \%$ des mères sans niveau. Cette fréquence était très élevée en Afghanistan avec $92,3 \%(13)$.

Les ménagères représentaient $44 \%$ de notre échantillon, ce résultat est similaire à celui de (2) [2] [2] qui avait trouvé $41 \%$ de ménagères dans la ville de Kinshasa et inférieur à celui trouvé par NANTOUME I, qui avait trouvé $79,3 \%$ de ménagère chez des mères des enfants de 6-59 mois dépistés MAM en 2017 à Yirimadio.

Connaissances, attitudes et comportements des mères :

La présente étude montre que $93 \%$ des mères avaient entendu parler de la malnutrition par carence et $45,4 \%$ pensaient que la maigreur est le premier signe clinique évocateur de la malnutrition par carence. Ce résultat est différent de celui que trouve NANTOUME I, de 38,1\% pour le manque d'appétit comme signe clinique à l'admission (10). La connaissance de la malnutrition par les mères est importante. En effet, certaines études ont établi le lien entre la malnutrition et le dysfonctionnement immunitaire de l'enfant (14). Ce dysfonctionnement peut aussi être la conséquence de la malnutrition (14). Au Ghana, Donkor et al. montre l'importance de la formation dans la communauté ne serait-ce que sur la réduction du stress lié au nombre de repas (15). Il en est de même, Frozanfar et al. montre la réduction de la prévalence de la malnutrition avec l'augmentation du niveau d'éducation (13). Nous voyons l'importance de la connaissance et de la formation sur les parents. Elles sont aussi importantes sur les prestataires de soins pour les enfants comme le prouve Chidziwisano au Malawi (16). Jacob Arriola et al. trouve qu'il est important de tenir compte des connaissances locales pour proposer des interventions pertinentes dans la lutte contre la malnutrition (5).

Dans notre étude, la quasi-totalité des répondants était consciente et connaissait bien les méfaits liés à la présence des ordures ménagères sur la santé humaine. Ce résultat est similaire à celui de AFRICARE (17), qui trouve $92 \%$ de répondants conscients.

Parmi les méfaits évoqués, dans notre étude $54,2 \%$ ont évoqué les maladies, il s'agit notamment du paludisme, la fièvre thyroïdes, la diarrhée, etc. Ce résultat est inférieur à celui de I'USAID (3), qui rapporte $66,6 \%$ de maladie comme conséquence de la mauvaise gestion des ordures ménagères.

Dans notre étude nous avons trouvé que $62 \%$ des mères utilisent la poubelle pour stocker leurs ordures ménagères et $38 \%$ n'utilisent pas de poubelles. Ce résultat est similaire à celui de AFRICARE (17), qui trouve que, $63 \%$ utilisent des poubelles pour stocker leurs ordures (sceau, sac, sachet), $37 \%$ des ménages n'utilisent pas des poubelles. II convient de souligner que la plupart des ménages utilisent des poubelles qui ne remplissent pas des normes d'hygiène.

En rapport avec l'évacuation des déchets, nous avons noté que la population de notre aire de sante utilisait la voie publique dans $94 \%$ des cas (rejet dans la rue, dans les décharges sauvages, dans les cours d'eau, dans les ravins, dans les caniveaux, terrain vague). II s'agit d'acte d'incivisme. En effet les ménages doivent utiliser les poubelles dont le contenu doit être ramassé régulièrement par les groupements d'intérêt économique (GIE) œuvrant dans ce domaine. II s'agit de la collecte primaire, vers les décharges publiques.

L'incinération à ciel ouvert était utilisée dans $6 \%$ de cas. Par rapport à l'incinération, nos résultats diffèrent avec ceux de l'étude menée au Niger sur la gestion des déchets d'élevage et ménager biodégradable. D'après cette étude, l'incinération à ciel ouvert était pratiquée dans $16,4 \%$ des cas et la voie publique dans $50 \%$ (4).

Comme moyen d'évacuation des eaux usées, $59 \%$ des enquêtées versaient les eaux usées dans les rues. $\mathrm{Ce}$ résultat est similaire à celui trouvé dans le quartier Doukoure par Dongo K et al. (18). II trouve de nombreuses insuffisances dans le système de gestion de l'assainissement des quartiers précaires, exposant les populations aux maladies liées à l'assainissement telles que le paludisme et la diarrhée (18). De Vita et al. et Gizaw et Worku trouvent une association significative entre les mauvaises conditions d'hygiène, d'eau et d'assainissement (WASH) et la malnutrition $(9,19,20)$. En Afghanistan, plus de la moitié des ménages avait des mauvaises conditions d'hygiène, d'eau et d'assainissement (13). Les bonnes conditions de WASH améliorent la morbidité liée à la malnutrition chez les enfants de 6-59 mois en Ethiopie (21).

\section{Conclusion}

Cette étude a montré que $62 \%$ des ménages possédaient des ouvrages d'assainissement tel que les poubelles des stockages des ordures. Le rejet dans les caniveaux ou sur les chaussés était le mode d'élimination utilisé par les ménages ; respectivement $37 \%, 27 \%, 26 \%$ soit un total de $94 \%$ des ménages. Ces comportements ne répondaient pas aux normes en matière de gestion des ordures. Dans notre étude, $59 \%$ des ménages déversaient directement les eaux usées dans la rue. Et la population (93\%) est consciente des dangers des ordures ménagères sur la santé humaine. Ces résultats montrent que la situation de l'assainissement est préoccupante à Yirimadio. Cette situation nécessite des solutions efficaces et durables, 
d'où la nécessité de la mise en place des stratégies pour une gestion durable des ordures ménagères dans le quartier de Yirimadio incluant l'information et la sensibilisation.

\section{Références}

1. Cellule de Planification et de Statistique du Ministère de la Santé, Direction Nationale de la Statistique et de l'Informatique, Ministère de l'Économie, de l'Industrie et du Commerce. Enquête Démographique et de Santé du Mali (EDSM-IV) 2006 [Internet]. Macro International Inc. Calverton, Maryland, USA; 2007 [cité 24 déc 2017]. Disponible sur:

https://www.dhsprogram.com/pubs/pdf/FR199/FR199.pdf 2. OMS. Malnutrition centre media, Aide-mémoire mai 2017 [Internet]. Genève, Suisse: OMS; 2017 [cité 19 août 2017]. Disponible sur:

http://www.who.int/nutrition/topics/moderate malnutrition/fr/

3. USAID. Présentation de la stratégie pour la période 2006-2010. USAID Mali; 2006.

4. Ag Iknane A, Konaté H, Diallo TF, Diarra M, et al. Module de formation en nutrition humaine, volume 1. Ministère de la Santé et Helen Keller International; 2005. 5. Jacob Arriola KR, Ellis A, Webb-Girard A, Ogutu EA, McClintic E, Caruso B, et al. Designing integrated interventions to improve nutrition and WASH behaviors in Kenya. Pilot Feasibility Stud [Internet]. 3 févr 2020 [cité 18 janv 2021];6. Disponible sur:

https://www.ncbi.nlm.nih.gov/pmc/articles/PMC6998333/

6. CSRéf de la Commune VI. Programme de Développement Sanitaire et social de Cercle (PDSC) 2013-2017. Bamako, Mali: District Sanitaire; 2012 p. 144. 7. GUITEYE A. Evaluation de la qualité de la prise en charge des consultations prénatales au centre de santé communautaire de Yirimadio en commune $\mathrm{VI} d u$ district de Bamako [Thèse de Médecine]. [Bamako]: USTTB; 2013.

8. Institut National de la Statistique (INSTAT). Enquête Nutritionnelle Anthropométrique et de Mortalité rétrospective, Mali, SMART 2016 [Internet]. Bamako: INSTAT; 2016 juill [cité 11 mai 2019] p. 117. Disponible sur: http://www.instat-

mali.org/contenu/eq/rafsmart16_eq.pdf

9. De Vita MV, Scolfaro C, Santini B, Lezo A, Gobbi F, Buonfrate D, et al. Malnutrition, morbidity and infection in the informal settlements of Nairobi, Kenya: an epidemiological study. Ital J Pediatr [Internet]. 14 janv 2019 [cité 18 janv 2021];45. Disponible sur:

https://www.ncbi.nlm.nih.gov/pmc/articles/PMC6332593/

10. NANTOUME I. Evaluation de la prise en charge de la malnutrition aiguë modérée chez les enfants de 0659 mois par les agents de santé communautaire de Yirimadio dans le district de Bamako [Thèse de Médecine]. [Bamako]: USTTB; 2018.

11. KEMESSO B. Evaluation de la prise en charge de la malnutrition aiguë sévère chez les enfants de 0 à 59mois admis dans le centre de santé communautaire (CSCOM) de Yirimadio [Thèse de Médecine]. [Bamako]: USTTB; 2016.

12. DJIRE A. Prise en charge des enfants de 6-59 mois en matière de malnutrition dans les cscom de la commune V [Thèse de Médecine]. [Bamako]: USTTB; 2013.

13. Frozanfar MK, Yoshida Y, Yamamoto E, Reyer JA, Dalil S, Rahimzad AD, et al. Acute malnutrition among under-five children in Faryab, Afghanistan: prevalence and causes. Nagoya J Med Sci. févr 2016;78(1):41-53.

14. Bourke CD, Berkley JA, Prendergast AJ. Immune Dysfunction as a Cause and Consequence of Malnutrition. Trends Immunol. juin 2016;37(6):386-98. 15. Donkor CM, Lee J, Lelijveld N, Adams M, Baltussen MM, Nyante GG, et al. Improving nutritional status of children with Cerebral palsy: a qualitative study of caregiver experiences and community-based training in Ghana. Food Sci Nutr. 15 nov 2018;7(1):35-43.

16. Chidziwisano K, Slekiene J, Mosler H-J, Morse T. Improving Complementary Food Hygiene Behaviors Using the Risk, Attitude, Norms, Ability, and SelfRegulation Approach in Rural Malawi. Am J Trop Med Hyg. mai 2020;102(5):1104-15.

17. AFRICARE. Rapports des indicateurs de l'ONG AFRICARE. Bamako: ONG AFRICARE; 2007 p. 63.

18. Dongo K, Kouamé FK, Koné B, Biém J, Tanner $\mathrm{M}$, Cissé $\mathrm{G}$. Analyse de la situation de l'environnement sanitaire des quartiers défavorisés dans le tissu urbain de Yopougon a Abidjan, Côte d'Ivoire. VertigO - Rev Électronique En Sci Environ [Internet]. 8 janv 2009 [cité 18 janv 2021];(Volume 8 Numéro 3). Disponible sur: http://journals.openedition.org/vertigo/6252

\section{Gizaw Z, Woldu W, Bitew BD. Acute}

malnutrition among children aged 6-59 months of the nomadic population in Hadaleala district, Afar region, northeast Ethiopia. Ital J Pediatr [Internet]. 7 févr 2018 [cité 18 janv 2021];44. Disponible sur: https://www.ncbi.nlm.nih.gov/pmc/articles/PMC5803907/ 20. van Cooten MH, Bilal SM, Gebremedhin S,

Spigt M. The association between acute malnutrition and water, sanitation, and hygiene among children aged 659 months in rural Ethiopia. Matern Child Nutr [Internet]. 1 juill 2018 [cité 18 janv 2021];15(1). Disponible sur: https://www.ncbi.nlm.nih.gov/pmc/articles/PMC7232102/ 21. Wasihun AG, Dejene TA, Teferi M, Marugán J, Negash L, Yemane D, et al. Risk factors for diarrhoea and malnutrition among children under the age of 5 years in the Tigray Region of Northern Ethiopia. PLOS ONE [Internet]. 26 nov 2018 [cité 18 janv 2021];13(11). Disponible sur: https://www.ncbi.nlm.nih.gov/pmc/articles/PMC6257922/ 
Liste des tableaux et figures :

Tableau I : Distribution des mères selon les caractéristiques sociodémographiques

\begin{tabular}{|c|c|c|}
\hline & Effectif $(\mathrm{N}=216)$ & $\%$ \\
\hline \multicolumn{3}{|c|}{ Classes d'âge en années } \\
\hline$[18-26[$ & 60 & 27,8 \\
\hline$[26-34[$ & 65 & 30,1 \\
\hline$[34-42[$ & 43 & 19,9 \\
\hline$[42-50[$ & 48 & 22,2 \\
\hline \multicolumn{3}{|c|}{ Durée de résidence dans le quartier en années } \\
\hline$[1-4[$ & 95 & 44,0 \\
\hline$[4-7[$ & 39 & 18,1 \\
\hline$[7-10[$ & 30 & 13,9 \\
\hline$[10-13[$ & 9 & 4,2 \\
\hline$[13-17[$ & 13 & 6,0 \\
\hline$[17-20[$ & 30 & 13,9 \\
\hline \multicolumn{3}{|c|}{ Statut matrimonial } \\
\hline Célibataire & 26 & 12,0 \\
\hline Mariée & 112 & 51,9 \\
\hline Veuve & 35 & 16,2 \\
\hline Divorcée & 43 & 19,9 \\
\hline \multicolumn{3}{|c|}{ Niveau d'instruction } \\
\hline Non scolarisée & 109 & 50,5 \\
\hline Primaire & 43 & 19,9 \\
\hline Secondaire & 31 & 14,4 \\
\hline Alphabétisée & 23 & 10,6 \\
\hline Universitaire & 10 & 4,6 \\
\hline \multicolumn{3}{|l|}{ Profession } \\
\hline Fonctionnaire & 17 & 7,9 \\
\hline Commerce & 91 & 42,1 \\
\hline Ménagère & 95 & 44,0 \\
\hline Autres & 13 & 06,0 \\
\hline
\end{tabular}

Tableau II : Répartition des mères selon les connaissances, attitudes et comportements

\begin{tabular}{lcc}
\hline & Effectif (N=216) & $\%$ \\
Signes cliniques de la malnutrition & 98 & 45,4 \\
\hline Maigreur & 60 & 27,8 \\
Manque d'appétit & 35 & 16,2 \\
Fébricule & 23 & 10,6 \\
Diarrhée & 20 & \\
Conséquences de la mauvaise gestion des ordures & ménagères sur la santé & 9,3 \\
Mauvaise odeur & 79 & 36,5 \\
Prolifération des vecteurs & 117 & 54,2 \\
Maladies & 56 & 25,9 \\
Moyens utilisés pour éliminer les ordures ménagères & 26,9 \\
Versement dans des caniveaux & 58 & 37,0 \\
Versement sur la route & 80 & 6,0 \\
Versement dans la rue & 13 & 4,2 \\
Terrain vagues & 9 & \\
Incinération & 127 & 58,8 \\
Moyens utilisés pour éliminer les eaux usées & 24 & 11,1 \\
Evacuation dans la rue & 65 & 30,1 \\
\hline Utilisation des fosses septiques & & \\
Utilisation des terrains vagues & & \\
\hline
\end{tabular}

